TRANSACTIONS OF THE

AMERICAN MATHEMATICAL SOCIETY

Volume 360, Number 11, November 2008, Pages 6089-6102

S 0002-9947(08)04507-8

Article electronically published on April 25, 2008

\title{
LIMIT THEOREMS FOR FREE MULTIPLICATIVE CONVOLUTIONS
}

\author{
HARI BERCOVICI AND JIUN-CHAU WANG
}

\begin{abstract}
We determine the distributional behavior for products of free random variables in a general infinitesimal triangular array. The main theorems in this paper extend a result for measures supported on the positive half-line, and provide a new limit theorem for measures on the unit circle with nonzero first moment.
\end{abstract}

\section{INTRODUCTION}

Given two probability measures $\mu, \nu$ on $\mathbb{R}_{+}=(0,+\infty)$, we will denote by $\mu \circledast \nu$ their classical multiplicative convolution, and by $\mu \otimes \nu$ their free multiplicative convolution. Thus, $\mu \circledast \nu$ is the distribution of $X Y$, where $X$ and $Y$ are classically independent positive random variables with distributions $\mu$ and $\nu$, respectively. Analogously, $\mu \otimes \nu$ is the distribution of $X^{1 / 2} Y X^{1 / 2}$, where $X$ and $Y$ are freely independent positive random variables with distributions $\mu$ and $\nu$. A triangular array $\left\{\nu_{n k}: n \geq 1,1 \leq k \leq k_{n}\right\}$ of probability measures on $\mathbb{R}_{+}$is said to be infinitesimal if

$$
\lim _{n \rightarrow \infty} \max _{1 \leq k \leq k_{n}} \nu_{n k}\left(\left\{t \in \mathbb{R}_{+}:|t-1| \geq \varepsilon\right\}\right)=0,
$$

for every $\varepsilon>0$. Given such an array, one is interested in the asymptotic behavior of the measures

$$
\mu_{n}=\nu_{n 1} \circledast \nu_{n 2} \circledast \cdots \circledast \nu_{n k_{n}}
$$

and

$$
\nu_{n}=\nu_{n 1} \otimes \nu_{n 2} \otimes \cdots \otimes \nu_{n k_{n}} .
$$

The case of $\mu_{n}$ is completely understood and is reduced to the theory of addition of independent random variables by a logarithmic change of variables. However, the free case $\nu_{n}$ does not simply reduce to the additive theory by this change of variables.

The problem was first addressed in 4, where a triangular array such that $\nu_{n 1}=\nu_{n 2}=\cdots=\nu_{n k_{n}}$ for all $n$ was considered. In this case, necessary and sufficient conditions were found for the weak convergence of the measures $\nu_{n}$. In particular, it was shown that the sequence $\nu_{n}$ converges weakly if $\mu_{n}$ converges, but not conversely.

In this paper we will find necessary and sufficient conditions for the weak convergence of $\nu_{n}$ without any further assumptions on the infinitesimal array. We

Received by the editors December 20, 2006.

2000 Mathematics Subject Classification. Primary 46L54; Secondary 60F05.

The first author was supported in part by a grant from the National Science Foundation.

(C)2008 American Mathematical Society 
also prove analogous results for infinitesimal triangular arrays on the unit circle $\mathbb{T}=\{z \in \mathbb{C}:|z|=1\}$. In both cases the possible limit of $\nu_{n}$ is $\bigotimes$-infinitely divisible as shown in [8] and [2].

The additive version of our results was studied earlier. Thus, consider an array $\left\{\mu_{n k}: n \geq 1,1 \leq k \leq k_{n}\right\}$ of probability measures on $\mathbb{R}$. Infinitesimality in this case means that

$$
\lim _{n \rightarrow \infty} \max _{1 \leq k \leq k_{n}} \mu_{n k}(\{t \in \mathbb{R}:|t| \geq \varepsilon\})=0, \quad \varepsilon>0 .
$$

Denote by

$$
\lambda_{n}=\mu_{n 1} * \mu_{n 2} * \cdots * \mu_{n k_{n}}
$$

the classical additive convolutions, and by

$$
\rho_{n}=\mu_{n 1} \boxplus \mu_{n 2} \boxplus \cdots \boxplus \mu_{n k_{n}}
$$

the free additive convolutions of these measures. When $\mu_{n 1}=\mu_{n 2}=\cdots=\mu_{n k_{n}}$ for all $n$, it was shown in 3] that $\lambda_{n}$ converges weakly if and only if $\rho_{n}$ converges weakly. This result was extended to arbitrary infinitesimal arrays by Chistyakov and Götze in [9]. These authors made heavy use of analytic subordination (first proved for the free additive convolution in [11] generically and in 7] for the general case; cf. also [12, 13, [1] and [8] for different approaches). Our methods do not require analytic subordination and are close to the original approach in 44.

The remainder of this paper is organized as follows. In Section 2, we describe the analytical apparatus necessary for the calculation of free multiplicative con-

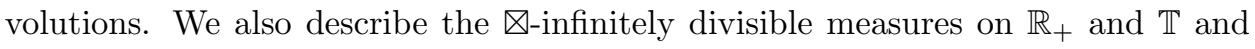
some useful approximation results. In Section 3 we give the convergence criteria for arrays on $\mathbb{R}_{+}$, and in Section 4 , we prove the analogous result for $\mathbb{T}$.

\section{Preliminaries}

The analogue of the Fourier transform for multiplicative free convolutions was discovered by Voiculescu [10] (see also [5, 6]). Denote by $\mathcal{M}_{+}$the collection of Borel probability measures defined on $\mathbb{R}_{+}$, and by $\mathcal{M}_{\mathbb{T}}^{\times}$the Borel probability measures $\nu$ supported on the circle $\mathbb{T}$ with nonzero first moment, i.e. $\int_{\mathbb{T}} t d \nu(t) \neq 0$.

Given $\nu \in \mathcal{M}_{+}$, one defines the analytic function $\psi_{\nu}$ by

$$
\psi_{\nu}(z)=\int_{0}^{\infty} \frac{t z}{1-t z} d \nu(t), \quad z \in \mathbb{C} \backslash(0,+\infty) .
$$

The function $\psi_{\nu}$ is univalent in the left half-plane $i \mathbb{C}^{+}$, and $\psi_{\nu}\left(i \mathbb{C}^{+}\right)$is a region contained in the circle with diameter $(-1,0)$; moreover, $\psi_{\nu}\left(i \mathbb{C}^{+}\right) \cap(-\infty, 0)=(-1,0)$. Setting $\Omega_{\nu}=\psi_{\nu}\left(i \mathbb{C}^{+}\right)$, one defines the $S$-transform of the measure $\nu$ to be

$$
S_{\nu}(z)=\frac{1+z}{z} \psi_{\nu}^{-1}(z), \quad z \in \Omega_{\nu} .
$$

The remarkable property of the $S$-transform is that for $\mu, \nu \in \mathcal{M}_{+}$, one has

$$
S_{\mu \bowtie \nu}(z)=S_{\mu}(z) S_{\nu}(z),
$$

for every $z$ in a neighborhood of $(-1,0)$.

For $\nu \in \mathcal{M}_{\mathbb{T}}^{\times}$, the function $\psi_{\nu}$ is defined by the formula given above (with the integral calculated over $\mathbb{T}$ ), but its domain of definition is now the open unit disk $\mathbb{D}=\{z \in \mathbb{C}:|z|<1\}$. The function $\psi_{\nu}$ has an inverse in a neighborhood of zero since $\psi_{\nu}^{\prime}(0)=\int_{\mathbb{T}} t d \nu(t) \neq 0$. The corresponding $S$-transform is defined in 
a neighborhood of zero. It is sometimes convenient to use a variation of the $S$ transform:

$$
\Sigma_{\nu}(z)=S_{\nu}\left(\frac{z}{1-z}\right) .
$$

If $\nu \in \mathcal{M}_{\mathbb{T}}^{\times}$, the function $\Sigma_{\nu}$ is also defined in a neighborhood of zero, and

$$
\Sigma_{\mu 凶 \nu}(z)=\Sigma_{\mu}(z) \Sigma_{\nu}(z), \quad \mu, \nu \in \mathcal{M}_{\mathbb{T}}^{\times},
$$

for all $z$ in a neighborhood of zero where all functions involved are defined.

The weak convergence of probability measures can be translated in terms of their $S$-transforms.

Theorem 2.1 ([], [6] $)$. (1) Given $\left\{\nu_{n}\right\}_{n=1}^{\infty} \subset \mathcal{M}_{+}$, the sequence $\left\{\nu_{n}\right\}_{n=1}^{\infty}$ converges weakly to a measure $\nu \in \mathcal{M}_{+}$if and only if there exist two positive numbers $0<b<a<1$ such that the disk $D$ with the diameter $(-a,-b)$ is contained in $\Omega_{\nu_{n}}$ for all $n$, and the sequence $\left\{S_{\nu_{n}}\right\}_{n=1}^{\infty}$ converges uniformly on $D$ to a function $S$.

(2) Given $\left\{\nu_{n}\right\}_{n=1}^{\infty} \subset \mathcal{M}_{\mathbb{T}}^{\times}$, the sequence $\left\{\nu_{n}\right\}_{n=1}^{\infty}$ converges weakly to a measure $\nu \in \mathcal{M}_{\mathbb{T}}^{\times}$if and only if there exists a neighborhood of zero $K \subset \mathbb{D}$ such that for all $\Sigma_{\nu_{n}}$ defined in $K$, the sequence $\left\{\Sigma_{\nu_{n}}\right\}_{n=1}^{\infty}$ converges uniformly on $K$ to a function $\Sigma$.

Moreover, if (1) is satisfied, then $S=S_{\nu}$, and if (2) is satisfied, then $\Sigma=\Sigma_{\nu}$.

An array $\left\{\nu_{n k}\right\}_{n, k} \subset \mathcal{M}_{\mathbb{T}}^{\times}$is infinitesimal if

$$
\lim _{n \rightarrow \infty} \max _{1 \leq k \leq k_{n}} \nu_{n k}(\{t \in \mathbb{T}:|\arg t| \geq \varepsilon\})=0,
$$

for every $\varepsilon>0$; here the principal value of the argument is used. The following proposition gives an approximation of the $S$-transform (see Theorem 3.1 in [4] and Theorem 1.1 and Theorem 2.1 in [2]).

Proposition 2.2. For $0<b<a<1$ and $\varepsilon \in(0,1)$, define $\bar{D}$ to be the closed disk with diameter $[-a,-b]$, and set $K_{\varepsilon}=\{z \in \mathbb{C}:|z| \leq \varepsilon\}$.

(1) If an array $\left\{\nu_{n k}\right\}_{n, k} \subset \mathcal{M}_{+}$is infinitesimal, then the functions $S_{\nu_{n k}}$ are defined in $\bar{D}$ for sufficiently large $n$, and we have

$$
S_{\nu_{n k}}(z)=1+\left[\int_{0}^{\infty} \frac{1-t}{1+z-t z} d \nu_{n k}(t)\right]\left(1+u_{n k}(z)\right)
$$

for all $z \in \bar{D}$, where $\lim _{n \rightarrow \infty} \max _{1 \leq k \leq k_{n}}\left|u_{n k}(z)\right|=0$ uniformly on $\bar{D}$.

(2) If an array $\left\{\nu_{n k}\right\}_{n, k} \subset \mathcal{M}_{\mathbb{T}}^{\times}$is infinitesimal, then $S_{\nu_{n k}}$ are defined in $K_{\varepsilon}$ when $n$ is large, and we have

$$
S_{\nu_{n k}}(z)=1+\left[\int_{\mathbb{T}} \frac{1-t}{1+z-t z} d \nu_{n k}(t)\right]\left(1+v_{n k}(z)\right),
$$

for all $z \in K_{\varepsilon}$, where $\lim _{n \rightarrow \infty} \max _{1 \leq k \leq k_{n}}\left|v_{n k}(z)\right|=0$ uniformly on $K_{\epsilon}$.

A measure $\nu \in \mathcal{M}_{+}$is said to be $\circledast$-infinitely divisible if, for each $n \in \mathbb{N}$, there exists a measure $\nu_{n} \in \mathcal{M}_{+}$such that

$$
\nu=\underbrace{\nu_{n} \circledast \nu_{n} \circledast \cdots \circledast \nu_{n}}_{n \text { times }} .
$$

The notion of $\otimes$-infinite divisibility is defined analogously. The study of $\circledast$-infinitely divisible measures on $\mathbb{R}_{+}$reduces (by a change of variable) to the study of the usual 
*-infinitely divisible measures on $\mathbb{R}$. The Fourier transform needs to be replaced by the Mellin-Fourier transform of a measure $\nu \in \mathcal{M}_{+}$defined by

$$
\Phi_{\nu}(s)=\int_{0}^{\infty} t^{i s} d \nu(t), \quad s \in \mathbb{R} .
$$

The fundamental property of the Mellin-Fourier transform is that

$$
\Phi_{\mu \circledast \nu}(s)=\Phi_{\mu}(s) \Phi_{\nu}(s) .
$$

A $\circledast$-infinitely divisible measure $\nu \in \mathcal{M}_{+}$has the Mellin-Fourier transform

$$
\Phi_{\nu}(s)=\exp \left[i \lambda s+\int_{0}^{\infty}\left(t^{-i s}-1+\frac{i s \log t}{\log ^{2} t+1}\right) \frac{\log ^{2} t+1}{\log ^{2} t} d \rho(t)\right], \quad s \in \mathbb{R},
$$

where $\lambda \in \mathbb{R}$ and $\rho$ is a finite positive Borel measure on $\mathbb{R}_{+}$. We use the notation $\nu_{\circledast}^{\lambda, \rho}$ to denote the $\circledast$-infinitely divisible measure determined by $\lambda$ and $\rho$. For $\otimes$ infinite divisibility we have the following formulas as in [5, 6]. A measure $\nu \in \mathcal{M}_{+}$ is $\nabla$-infinitely divisible if and only if there exist $\gamma \in \mathbb{R}$ and a finite positive Borel measure $\sigma$ on the compact space $[0,+\infty]$ such that $S_{\nu}(z)=\exp \left(v_{\gamma, \sigma}(z)\right)$, where $v_{\gamma, \sigma}$ is given by

$$
v_{\gamma, \sigma}\left(\frac{z}{1-z}\right)=\gamma-\sigma(\{+\infty\}) z+\int_{[0,+\infty)} \frac{1+t z}{z-t} d \sigma(t), \quad z \in \mathbb{C} \backslash[0,1] .
$$

A measure $\nu \in \mathcal{M}_{\mathbb{T}}^{\times}$is $\bigotimes$-infinitely divisible if and only if there exist $\gamma \in \mathbb{R}$ and a finite positive Borel measure $\sigma$ on $\mathbb{T}$ such that $\Sigma_{\nu}(z)=\exp \left(u_{\gamma, \sigma}(z)\right)$, where $u_{\gamma, \sigma}$ is given by

$$
u_{\gamma, \sigma}(z)=-i \gamma+\int_{\mathbb{T}} \frac{1+t z}{1-t z} d \sigma(t), \quad z \in \mathbb{D} .
$$

We denote by $\nu_{\otimes}^{\gamma, \sigma}$ the $\bigotimes$-infinitely divisible measure determined by $\gamma$ and $\sigma$. There is a unique $\bigotimes$-infinitely divisible measure $m$ on $\mathbb{T}$ such that its first moment is zero. This is the Haar, or normalized arclength measure.

We conclude this section with a result which will be used repeatedly.

Lemma 2.3. Consider a sequence $\left\{r_{n}\right\}_{n=1}^{\infty} \subset \mathbb{R}$ and two triangular arrays $\left\{z_{n k}\right.$ : $\left.n \geq 1,1 \leq k \leq k_{n}\right\},\left\{w_{n k}: n \geq 1,1 \leq k \leq k_{n}\right\}$ of complex numbers. Assume that

(1) $\Im w_{n k} \geq 0$, for $n \geq 1$ and $1 \leq k \leq k_{n}$.

(2) $z_{n k}=w_{n k}\left(1+\varepsilon_{n k}\right)$, where

$$
\varepsilon_{n}=\max _{1 \leq k \leq k_{n}}\left|\varepsilon_{n k}\right|
$$

converges to zero as $n \rightarrow \infty$.

(3) There exists a positive constant $M$ such that for sufficiently large $n$,

$$
\left|\Re w_{n k}\right| \leq M \Im w_{n k} .
$$

Then the sequence $\left\{r_{n}+\sum_{k=1}^{k_{n}} z_{n k}\right\}_{n=1}^{\infty}$ converges if and only if the sequence $\left\{r_{n}+\right.$ $\left.\sum_{k=1}^{k_{n}} w_{n k}\right\}_{n=1}^{\infty}$ converges. Moreover, the two sequences have the same limit.

Proof. The assumptions on $\left\{z_{n k}\right\}_{n, k}$ and $\left\{w_{n k}\right\}_{n, k}$ imply

$$
\left|\left(r_{n}+\sum_{k=1}^{k_{n}} z_{n k}\right)-\left(r_{n}+\sum_{k=1}^{k_{n}} w_{n k}\right)\right| \leq 2(1+M) \varepsilon_{n}\left(\sum_{k=1}^{k_{n}} \Im w_{n k}\right)
$$


and

$$
\left(1-\varepsilon_{n}-M \varepsilon_{n}\right)\left(\sum_{k=1}^{k_{n}} \Im w_{n k}\right) \leq\left|\sum_{k=1}^{k_{n}} \Im z_{n k}\right|,
$$

for sufficiently large $n$. If the sequence $\left\{r_{n}+\sum_{k=1}^{k_{n}} z_{n k}\right\}_{n=1}^{\infty}$ converges to a complex number $z$, (2.2) implies that $\left\{\sum_{k=1}^{k_{n}} \Im w_{n k}\right\}_{n=1}^{\infty}$ is bounded, and then (2.1) shows that the sequence $\left\{r_{n}+\sum_{k=1}^{k_{n}} w_{n k}\right\}_{n=1}^{\infty}$ also converges to $z$. Conversely, if $\left\{r_{n}+\right.$ $\left.\sum_{k=1}^{k_{n}} w_{n k}\right\}_{n=1}^{\infty}$ converges to $z$, then the sequence $\left\{\sum_{k=1}^{k_{n}} \Im w_{n k}\right\}_{n=1}^{\infty}$ is bounded and hence by (2.1) the sequence $\left\{r_{n}+\sum_{k=1}^{k_{n}} z_{n k}\right\}_{n=1}^{\infty}$ converges to $z$ as well.

\section{Free multiplicative convolution on $\mathbb{R}_{+}$}

Given an infinitesimal triangular array $\left\{\nu_{n k}: 1 \leq k \leq k_{n}, n \in \mathbb{N}\right\} \subset \mathcal{M}_{+}$and $\tau>0$, define positive numbers

$$
b_{n k}=\exp \left(\int_{e^{-\tau}}^{e^{\tau}} \log t d \nu_{n k}(t)\right)
$$

and measures $\nu_{n k}^{\circ}$ by

$$
d \nu_{n k}^{\circ}(t)=d \nu_{n k}\left(b_{n k} t\right)
$$

Obviously, $\max _{1 \leq k \leq k_{n}}\left|b_{n k}-1\right| \rightarrow 0$ as $n \rightarrow \infty$, and hence the array $\left\{\nu_{n k}^{\circ}\right\}_{n, k}$ is also infinitesimal. Define

$$
g_{n k}(w)=\int_{0}^{\infty} \frac{t^{2}-1}{t^{2}+1} d \nu_{n k}^{\circ}\left(\frac{1}{t}\right)+\int_{0}^{\infty}\left[\frac{1+t w}{w-t}\right] \frac{(t-1)^{2}}{t^{2}+1} d \nu_{n k}^{\circ}\left(\frac{1}{t}\right),
$$

for $w \in \mathbb{C} \backslash[0,+\infty)$. Note that $g_{n k}(\bar{w})=\overline{g_{n k}(w)}$ and $\Im g_{n k}(w) \leq 0$ for all $w$ such that $\Im w>0$.

Lemma 3.1. For every compact set $K \subset \mathbb{C}^{+} \cap\left(i \mathbb{C}^{+}\right)$there exists a positive constant $M=M(\tau, K)$ such that for sufficiently large $n$, we have

$$
\left|\Re g_{n k}(w)\right| \leq M\left|\Im g_{n k}(w)\right|, \quad w \in K, 1 \leq k \leq k_{n} .
$$

Proof. We assume for convenience that $\tau=1$. No generality is lost since one can make a linear change of variable to modify the value of $\tau$. By a change of variable we have

$$
\int_{0}^{\infty} \frac{t^{2}-1}{t^{2}+1} d \nu_{n k}^{\circ}\left(\frac{1}{t}\right)=\int_{-\infty}^{\infty} \frac{1-e^{2 x}}{1+e^{2 x}} d \rho_{n k}\left(x+a_{n k}\right)
$$

and

$$
\int_{0}^{\infty} \frac{(t-1)^{2}}{t^{2}+1} d \nu_{n k}^{\circ}\left(\frac{1}{t}\right)=\int_{-\infty}^{\infty} \frac{\left(1-e^{x}\right)^{2}}{1+e^{2 x}} d \rho_{n k}\left(x+a_{n k}\right),
$$

where the probability measure $\rho_{n k}$ is defined as $d \rho_{n k}(x)=d \nu_{n k}\left(e^{x}\right)$, and $a_{n k}=$ $\int_{|x|<1} x d \rho_{n k}(x)$. Note that the family $\left\{\rho_{n k}\right\}_{n, k}$ is now an infinitesimal family of probability measures on $\mathbb{R}$, and hence

$$
\lim _{n \rightarrow \infty} \max _{1 \leq k \leq k_{n}}\left|a_{n k}\right|=0 .
$$


We proceed by rewriting

$$
\begin{aligned}
\int_{-\infty}^{\infty} \frac{1-e^{2 x}}{1+e^{2 x}} d \rho_{n k}\left(x+a_{n k}\right)= & \int_{|x|<1}\left[\frac{1-e^{2\left(x-a_{n k}\right)}}{1+e^{2\left(x-a_{n k}\right)}}+\left(x-a_{n k}\right)\right] d \rho_{n k}(x) \\
& -\int_{|x| \geq 1} a_{n k} d \rho_{n k}(x)+\int_{|x| \geq 1} \frac{1-e^{2\left(x-a_{n k}\right)}}{1+e^{2\left(x-a_{n k}\right)}} d \rho_{n k}(x) .
\end{aligned}
$$

It is easy to see that

$$
\left|1-e^{2\left(x-a_{n k}\right)}+\left(x-a_{n k}\right)+\left(x-a_{n k}\right) e^{2\left(x-a_{n k}\right)}\right| \leq 60\left(x-a_{n k}\right)^{2},
$$

for $|x|<1$. Consequently, for all $n$ and $k$ we have

$$
\left|\int_{|x|<1}\left[\frac{1-e^{2\left(x-a_{n k}\right)}}{1+e^{2\left(x-a_{n k}\right)}}+\left(x-a_{n k}\right)\right] d \rho_{n k}(x)\right| \leq 60 \int_{-\infty}^{\infty} \frac{\left(1-e^{x}\right)^{2}}{1+e^{2 x}} d \rho_{n k}\left(x+a_{n k}\right) .
$$

Since the family $\left\{\rho_{n k}\right\}_{n, k}$ is infinitesimal, there exists $N \in \mathbb{N}$ such that

$$
\left|a_{n k}\right| \leq \frac{1}{2}
$$

for all $n \geq N, 1 \leq k \leq k_{n}$. Note that

$$
\frac{\left(1-e^{x}\right)^{2}}{1+e^{2 x}} \geq \frac{(1-\sqrt{e})^{2}}{1+e} \quad \text { and } 5\left(1-e^{x}\right)^{2} \geq\left|1-e^{2 x}\right|,
$$

for all $|x| \geq \frac{1}{2}$. We deduce that for $n \geq N, 1 \leq k \leq k_{n}$ we have

$$
\begin{aligned}
\left|\int_{|x| \geq 1} a_{n k} d \rho_{n k}(x)\right| & \leq \int_{|x| \geq 1} d \rho_{n k}(x) \\
& \leq \frac{1+e}{(1-\sqrt{e})^{2}} \int_{-\infty}^{\infty} \frac{\left(1-e^{x}\right)^{2}}{1+e^{2 x}} d \rho_{n k}\left(x+a_{n k}\right)
\end{aligned}
$$

and

$$
\left|\int_{|x| \geq 1} \frac{1-e^{2\left(x-a_{n k}\right)}}{1+e^{2\left(x-a_{n k}\right)}} d \rho_{n k}(x)\right| \leq 5 \int_{-\infty}^{\infty} \frac{\left(1-e^{x}\right)^{2}}{1+e^{2 x}} d \rho_{n k}\left(x+a_{n k}\right) .
$$

Therefore, for sufficiently large $n$,

$$
\left|\int_{0}^{\infty} \frac{t^{2}-1}{t^{2}+1} d \nu_{n k}^{\circ}\left(\frac{1}{t}\right)\right| \leq 74 \int_{0}^{\infty} \frac{(t-1)^{2}}{t^{2}+1} d \nu_{n k}^{\circ}\left(\frac{1}{t}\right) .
$$

The compactness of the set $K$ implies the existence of positive constants $M_{1}$ and $M_{2}$ such that

$$
\left|\Re\left[\frac{1+t w}{w-t}\right]\right| \leq M_{1}
$$

and

$$
\left|\Im\left[\frac{1+t w}{w-t}\right]\right|=-\Im\left[\frac{1+t w}{w-t}\right] \geq M_{2}
$$


for all $t \in(0,+\infty)$ and $w \in K$. Hence, we have for sufficiently large $n$ and for $w \in K$,

$$
\begin{aligned}
\left|\Re g_{n k}(w)\right| & =\left|\int_{0}^{\infty} \frac{t^{2}-1}{t^{2}+1} d \nu_{n k}^{\circ}\left(\frac{1}{t}\right)+\int_{0}^{\infty} \Re\left[\frac{1+t w}{w-t}\right] \frac{(t-1)^{2}}{t^{2}+1} d \nu_{n k}^{\circ}\left(\frac{1}{t}\right)\right| \\
& \leq 74 \int_{0}^{\infty} \frac{(t-1)^{2}}{t^{2}+1} d \nu_{n k}^{\circ}\left(\frac{1}{t}\right)+M_{1} \int_{0}^{\infty} \frac{(t-1)^{2}}{t^{2}+1} d \nu_{n k}^{\circ}\left(\frac{1}{t}\right) \\
& \leq \frac{\left(74+M_{1}\right)}{M_{2}} \int_{0}^{\infty} M_{2} \frac{(t-1)^{2}}{t^{2}+1} d \nu_{n k}^{\circ}\left(\frac{1}{t}\right) \\
& \leq-\frac{\left(74+M_{1}\right)}{M_{2}} \int_{0}^{\infty} \Im\left[\frac{1+t w}{w-t}\right] \frac{(t-1)^{2}}{t^{2}+1} d \nu_{n k}^{\circ}\left(\frac{1}{t}\right) \\
& =\frac{74+M_{1}}{M_{2}}\left|\Im g_{n k}(w)\right| .
\end{aligned}
$$

The result follows with $M=\left(74+M_{1}\right) / M_{2}$.

Fix a closed disk $\bar{D} \subset i \mathbb{C}^{+}$with diameter $[-a,-b]$, where $0<b<a<1$. By Proposition 2.2, $S_{\nu_{n k}}$ is defined in $\bar{D}$ for large $n$. Setting $w=z /(1+z)$, and using the identity

$$
\frac{(w-1)(t-1)}{w-t}=\frac{t^{2}-1}{t^{2}+1}+\left[\frac{1+t w}{w-t}\right] \frac{(t-1)^{2}}{t^{2}+1},
$$

we see that the function $S_{\nu_{n k}^{\circ}}$ admits the following approximation:

$$
S_{\nu_{n k}^{\circ}}\left(\frac{w}{1-w}\right)-1=g_{n k}(w)\left(1+u_{n k}\left(\frac{w}{1-w}\right)\right)
$$

in another closed disk $\overline{D_{0}}=\{z /(1+z): z \in \bar{D}\}$ with real center, and

$$
\lim _{n \rightarrow \infty} \max _{1 \leq k \leq k_{n}}\left|u_{n k}\left(\frac{w}{1-w}\right)\right|=0,
$$

uniformly for all $w \in \overline{D_{0}}$. Note that

$$
S_{\nu_{n k}^{\circ}}\left(\frac{w}{1-w}\right)=b_{n k} S_{\nu_{n k}}\left(\frac{w}{1-w}\right) .
$$

The infinitesimality of the array $\left\{\nu_{n k}\right\}_{n, k}$ also shows that $S_{\nu_{n k}}(z)$ converges uniformly in $k$ and $z \in \bar{D}$ to 1 as $n \rightarrow \infty$; indeed, $S_{\delta_{1}} \equiv 1$. Hence, for sufficiently large $n$, the principal branch of $\log S_{\nu_{n k}}(z)$ is defined in $\bar{D}$. Furthermore, since

$$
\log w=w-1+o(|w-1|)
$$

as $w \rightarrow 1$, it is easy to see from Lemma 3.1 and Lemma 2.3 that we have the following result. Fix a real number $\gamma$, and a finite positive Borel measure $\sigma$ on $[0,+\infty]$.

Lemma 3.2. Let $\left\{\alpha_{n}\right\}_{n=1}^{\infty}$ be a sequence of positive real numbers. Then the sequence of functions $\left\{-\log \alpha_{n}+\sum_{k=1}^{k_{n}} \log S_{\nu_{n k}}(z)\right\}_{n=1}^{\infty}$ converges to $v_{\gamma, \sigma}(z)$ uniformly for all $z \in \bar{D}$ as $n \rightarrow \infty$ if and only if

$$
\lim _{n \rightarrow \infty}\left(-\log \alpha_{n}+\sum_{k=1}^{k_{n}}\left[g_{n k}(w)-\log b_{n k}\right]\right)=v_{\gamma, \sigma}\left(\frac{w}{1-w}\right)
$$

uniformly for all $w \in \overline{D_{0}}$. 
Theorem 3.3. For an infinitesimal array $\left\{\nu_{n k}\right\}_{n, k} \subset \mathcal{M}_{+}$and a sequence $\left\{\alpha_{n}\right\}_{n=1}^{\infty}$ $\subset \mathbb{R}_{+}$, the following two assertions are equivalent:

(1) The sequence $\nu_{n 1} \otimes \nu_{n 2} \otimes \cdots \otimes \nu_{n k_{n}} \otimes \delta_{\alpha_{n}}$ converges weakly to $\nu_{凶}^{\gamma, \sigma}$.

(2) The sequence of measures

$$
d \sigma_{n}(t)=\sum_{k=1}^{k_{n}} \frac{(t-1)^{2}}{t^{2}+1} d \nu_{n k}^{\circ}\left(\frac{1}{t}\right)
$$

converges weakly in $[0,+\infty]$ to $\sigma$, and the sequence

$$
\gamma_{n}=-\log \alpha_{n}+\sum_{k=1}^{k_{n}}\left[\int_{0}^{\infty} \frac{t^{2}-1}{t^{2}+1} d \nu_{n k}^{\circ}\left(\frac{1}{t}\right)-\log b_{n k}\right]
$$

converges to $\gamma$ as $n \rightarrow \infty$.

Proof. Assume (1) holds. From Theorem 2.1, there exists a closed disk $\bar{D}$ with real center such that

$$
\lim _{n \rightarrow \infty}\left(\frac{1}{\alpha_{n}} \prod_{k=1}^{k_{n}} S_{\nu_{n k}}(z)\right)=S_{\nu_{\searrow}^{\gamma, \sigma}}(z)=\exp \left(v_{\gamma, \sigma}(z)\right)
$$

uniformly on the disk $\bar{D}$. We may choose $\bar{D}$ small enough so that $\exp \left(v_{\gamma, \sigma}(z)\right)$ is in $-i \mathbb{C}^{+}$on $\bar{D}$. Applying the principal branch of the logarithm function, we deduce that

$$
\lim _{n \rightarrow \infty}\left(-\log \alpha_{n}+\sum_{k=1}^{k_{n}} \log S_{\nu_{n k}}(z)\right)=v_{\gamma, \sigma}(z),
$$

uniformly on $\bar{D}$. Thus, Lemma 3.2 implies that

$$
\lim _{n \rightarrow \infty}\left(-\log \alpha_{n}+\sum_{k=1}^{k_{n}}\left[g_{n k}(w)-\log b_{n k}\right]\right)=v_{\gamma, \sigma}\left(\frac{w}{1-w}\right)
$$

uniformly on $\overline{D_{0}}=\{z /(1+z): z \in \bar{D}\}$. Note that

$$
-\log \alpha_{n}+\sum_{k=1}^{k_{n}}\left[g_{n k}(w)-\log b_{n k}\right]=\gamma_{n}+\int_{0}^{\infty} \frac{1+t w}{w-t} d \sigma_{n}(t) .
$$

Considering the imaginary part of the equation (3.1), we have

$$
-\Im w \int_{[0,+\infty)} \frac{1+t^{2}}{|w-t|^{2}} d \sigma(t)=-\lim _{n \rightarrow \infty} \Im w \int_{(0,+\infty)} \frac{1+t^{2}}{|w-t|^{2}} d \sigma_{n}(t) .
$$

Note that the function $t \mapsto \frac{1+t^{2}}{|w-t|^{2}}$ is bounded away from zero and infinity for all $w \in \overline{D_{0}}$; moreover, if $\Im w \neq 0$, then (3.3) shows that

$$
\sup _{n} \sigma_{n}((0,+\infty))<+\infty
$$

and hence the family $\left\{\sigma_{n}\right\}_{n=1}^{\infty}$ has a weak cluster point $\sigma^{\prime}$ on the compact space $[0,+\infty]$. Then (3.3) shows that $\sigma^{\prime}=\sigma$, and consequently the measures $\sigma_{n}$ converge weakly to $\sigma$ on $[0,+\infty]$ as $n \rightarrow \infty$. Then it is easy to see from (3.1) and (3.2) that $\lim _{n \rightarrow \infty} \gamma_{n}=\gamma$.

Conversely, assume (2) holds. The infinitesimality of the array $\left\{\nu_{n k}\right\}_{n, k}$ implies that there exist $a^{\prime}, b^{\prime} \in(0,1)$ with $b^{\prime}<a^{\prime}$ such that $S_{\nu_{n k}}$ are defined in $\overline{D^{\prime}}$, the closed 
disk with diameter $\left[-a^{\prime},-b^{\prime}\right]$, for sufficiently large $n$. Let $\overline{D_{0}^{\prime}}=\left\{z /(1+z): z \in \overline{D^{\prime}}\right\}$ and observe that there exists a positive constant $M=M\left(a^{\prime}, b^{\prime}\right)$ such that

$$
\left|\frac{1+t w}{w-t}\right| \leq M, \quad w \in \overline{D_{0}^{\prime}}, t \in(0,+\infty) .
$$

Thus, in view of (3.2), we deduce that (3.1) holds pointwise in $\overline{D_{0}^{\prime}}$. Since $\Im g_{n k}(w) \leq$ 0 for $w \in D_{0}^{\prime} \cap i \mathbb{C}^{+}$, the family $\left\{-\log \alpha_{n}+\sum_{k=1}^{k_{n}}\left[g_{n k}(w)-\log b_{n k}\right]\right\}_{n=1}^{\infty}$ is normal in $D_{0}^{\prime} \cap i \mathbb{C}^{+}$. Moreover, note that $g_{n k}(\bar{w})=\overline{g_{n k}(w)}$ and

$$
v_{\gamma, \sigma}\left(\frac{\bar{w}}{1-\bar{w}}\right)=\overline{v_{\gamma, \sigma}\left(\frac{w}{1-w}\right)},
$$

for $w \in \overline{D_{0}^{\prime}}$. Therefore, as an application of Montel's theorem, we conclude that (3.1) holds uniformly on compact subsets of $\overline{D_{0}^{\prime}}$. From Lemma 3.2 we conclude that there exists a smaller closed disk $\overline{D^{\prime \prime}} \subset \overline{D^{\prime}}$ with real center, such that

$$
\lim _{n \rightarrow \infty}\left(-\log \alpha_{n}+\sum_{k=1}^{k_{n}} \log S_{\nu_{n k}}(z)\right)=v_{\gamma, \sigma}(z),
$$

uniformly on $\overline{D^{\prime \prime}}$. Applying the exponential, we obtain

$$
\lim _{n \rightarrow \infty}\left(\frac{1}{\alpha_{n}} \prod_{k=1}^{k_{n}} S_{\nu_{n k}}(z)\right)=S_{\nu_{\bigotimes}^{\gamma, \sigma}}(z)=\exp \left(v_{\gamma, \sigma}(z)\right)
$$

uniformly on $\overline{D^{\prime \prime}}$. Therefore (1) follows from Theorem 2.1.

It has been pointed out in 4 that the weak convergence criteria for products of free and independent random variables are not equivalent. Nevertheless, the following correspondence is true.

Corollary 3.4. Given an infinitesimal array $\left\{\nu_{n k}\right\}_{n, k} \subset \mathcal{M}_{+}$and a sequence $\left\{\alpha_{n}\right\}_{n=1}^{\infty} \subset \mathbb{R}_{+}$, the following two statements are equivalent:

(1) The sequence $\nu_{n 1} \otimes \nu_{n 2} \otimes \cdots \otimes \nu_{n k_{n}} \otimes \delta_{\alpha_{n}}$ converges weakly to $\nu_{\otimes}^{\gamma, \sigma}$ and $\sigma(\{0\})=\sigma(\{\infty\})=0$.

(2) The sequence $\nu_{n 1} \circledast \nu_{n 2} \circledast \cdots \circledast \nu_{n k_{n}} \circledast \delta_{\alpha_{n}}$ converges weakly to $\nu_{\circledast}^{\lambda, \rho}$.

If conditions (1) and (2) are satisfied, then the measures $\sigma$ and $\rho$ are related by

$$
d \sigma(t)=\frac{\log ^{2} t+1}{\log ^{2} t} \frac{(t-1)^{2}}{t^{2}+1} d \rho(t)
$$

and

$$
\gamma-\lambda=\int_{0}^{\infty}\left(\frac{t^{2}-1}{t^{2}+1}+\frac{\log ^{2} t}{\log ^{2} t+1}\right) \frac{\log ^{2} t+1}{\log ^{2} t} d \rho(t) .
$$

Proof. The proof is identical with that of Theorem 4.2 in [4].

\section{Free multiplicative COnVolution on $\mathbb{T}$}

Fix an infinitesimal array $\left\{\nu_{n k}: n \geq 1,1 \leq k \leq k_{n}\right\} \subset \mathcal{M}_{\mathbb{T}}^{\times}$and $\tau \in(0, \pi)$. Consider the centering constant

$$
b_{n k}=\exp \left(\int_{|\arg t|<\tau} \log t d \nu_{n k}(t)\right),
$$


and the centered measure $\nu_{n k}^{\circ}$ obtained as follows:

$$
d \nu_{n k}^{\circ}(t)=d \nu_{n k}\left(b_{n k} t\right)
$$

Here, as before, $\log t=i \arg t$ represents the principal branch of $\log t$. We have $\max _{1 \leq k \leq k_{n}}\left|\arg b_{n k}\right| \rightarrow 0$ as $n \rightarrow \infty$, and hence the array $\left\{\nu_{n k}^{\circ}\right\}_{n, k}$ is also infinitesimal. Define

$$
h_{n k}(z)=-i \int_{\mathbb{T}} \Im t d \nu_{n k}^{\circ}(t)+\int_{\mathbb{T}} \frac{1+t z}{1-t z}(1-\Re t) d \nu_{n k}^{\circ}(t), \quad z \in \mathbb{D} .
$$

Note that $\Re h_{n k}(z) \geq 0$ for all $z \in \mathbb{D}$.

Lemma 4.1. For every compact neighborhood of zero $K \subset \mathbb{D}$, there exists a positive constant $M=M(\tau, K)$ such that for sufficiently large $n$, we have

$$
\left|\Im h_{n k}(z)\right| \leq M \Re h_{n k}(z), \quad z \in K, 1 \leq k \leq k_{n} .
$$

Proof. We may again assume that $\tau=1$. Define probability measures $\rho_{n k}$ on $\mathbb{R}$ such that $\rho_{n k}(\sigma)=\nu_{n k}\left(e^{i \sigma}\right)$ if $\sigma \subset[-\pi, \pi)$, and $\rho_{n k}(\sigma)=0$ when $\sigma \cap[-\pi, \pi)=\phi$. Changing variables, we have

$$
\int_{\mathbb{T}} \Im t d \nu_{n k}^{\circ}(t)=\int_{-\infty}^{\infty} \sin x d \rho_{n k}\left(x+a_{n k}\right)
$$

and

$$
\int_{\mathbb{T}}(1-\Re t) d \nu_{n k}^{\circ}(t)=\int_{-\infty}^{\infty}(1-\cos x) d \rho_{n k}\left(x+a_{n k}\right),
$$

where $a_{n k}=\int_{|x|<1} x d \rho_{n k}(x)=\int_{|\arg t|<1} \arg t d \nu_{n k}(t)$. The infinitesimality of the array $\left\{\nu_{n k}\right\}_{n, k}$ implies that for sufficiently large $n$,

$$
\max _{1 \leq k \leq k_{n}}\left|a_{n k}\right| \leq \frac{1}{10} .
$$

By using the elementary inequalities

$$
|\sin x-x| \leq 2(1-\cos x), \quad-2 \leq x \leq 2,
$$

and

we have

$$
\frac{1}{10}+|\sin x| \leq 10(1-\cos x), \text { where } \frac{9}{10} \leq|x| \leq \pi+\frac{9}{10},
$$

$$
\begin{aligned}
\left|\int_{\mathbb{T}} \Im t d \nu_{n k}^{\circ}(t)\right| & \leq\left|\int_{|x|<1}\left[\sin \left(x-a_{n k}\right)-\left(x-a_{n k}\right)\right] d \rho_{n k}(x)\right| \\
& +\left|\int_{[-\pi,-1] \cup[1, \pi)} a_{n k} d \rho_{n k}(x)\right| \\
& +\left|\int_{[-\pi,-1] \cup[1, \pi)} \sin \left(x-a_{n k}\right) d \rho_{n k}(x)\right| \\
& \leq 12 \int_{\mathbb{T}}(1-\Re t) d \nu_{n k}^{\circ}(t),
\end{aligned}
$$

for large $n$ and $1 \leq k \leq k_{n}$. Also, from the compactness of the set $K$, there exist two positive constants $M_{1}$ and $M_{2}$ such that

$$
\left|\Re\left[\frac{1+t z}{1-t z}\right]\right|=\Re\left[\frac{1+t z}{1-t z}\right] \geq M_{1}
$$


and

$$
\left|\Im\left[\frac{1+t z}{1-t z}\right]\right| \leq M_{2}
$$

for all $t \in \mathbb{T}$ and $z \in K$. The result follows as in the proof of Lemma 3.1, with $M=\left(12+M_{2}\right) / M_{1}$.

Suppose $K \subset \mathbb{D}$ is a neighborhood of zero. The infinitesimality of the array $\left\{\nu_{n k}\right\}_{n . k}$ implies that $S_{\nu_{n k}}(z)$ converges uniformly in $k$ and $z \in K$ to 1 as $n \rightarrow \infty$, and hence for sufficiently large $n, \Sigma_{\nu_{n k}}(z)$ and the principal branch of $\log \Sigma_{\nu_{n k}}(z)$ are defined in $K^{\prime}=\{z /(1+z): z \in K\}$.

Fix a real number $\gamma$ and a finite positive Borel measure $\sigma$ on $\mathbb{T}$.

Lemma 4.2. Let $\left\{\lambda_{n}\right\}_{n=1}^{\infty} \subset \mathbb{T}$. Then

$$
\lim _{n \rightarrow \infty} \exp \left(-\log \lambda_{n}+\sum_{k=1}^{k_{n}} \log \Sigma_{\nu_{n k}}(z)\right)=\Sigma_{\nu_{凶}^{\gamma, \sigma}}(z)
$$

uniformly on $K^{\prime}$ if and only if

$$
\lim _{n \rightarrow \infty} \exp \left(-\log \lambda_{n}+\sum_{k=1}^{k_{n}}\left[h_{n k}(z)-\log b_{n k}\right]\right)=\Sigma_{\nu_{\unrhd}^{\gamma, \sigma}}(z)
$$

uniformly on $K^{\prime}$.

Proof. From Proposition 2.2, we have the following approximation for the function $S_{\nu_{n k}^{\circ}}^{\circ}$

$$
S_{\nu_{n k}^{\circ}}(z)=1+\left[\int_{\mathbb{T}} \frac{1-t}{1+z-t z} d \bar{\nu}_{n k}(t)\right]\left(1+v_{n k}(z)\right), \quad z \in K,
$$

where

$$
v_{n}(z)=\max _{1 \leq k \leq k_{n}}\left|v_{n k}(z)\right|
$$

satisfies $\lim _{n \rightarrow \infty} v_{n}(z)=0$ uniformly in $K$. Introducing a change of variable $z \mapsto$ $\frac{z}{1-z}$ and using the identity

$$
\frac{(1-t)(1-z)}{1-t z}=-i \Im t+\frac{1+t z}{1-t z}(1-\Re t),
$$

we conclude that

$$
b_{n k} \Sigma_{\nu_{n k}}(z)=\Sigma_{\nu_{n k}^{\circ}}(z)=1+h_{n k}(z)\left(1+v_{n k}\left(\frac{z}{1-z}\right)\right), \quad z \in K^{\prime} .
$$

Lemmas 4.1 and 2.3 imply that for any sequence of purely imaginary numbers $\left\{r_{n}\right\}_{n=1}^{\infty}$, the sequence $\left\{r_{n}+\sum_{k=1}^{k_{n}}\left[\Sigma_{\nu_{n k}^{\circ}}(z)-1\right]\right\}_{n=1}^{\infty}$ converges uniformly on $K^{\prime}$ if and only if the sequence $\left\{r_{n}+\sum_{k=1}^{k_{n}} h_{n k}(z)\right\}_{n=1}^{\infty}$ converges uniformly on $K^{\prime}$. Moreover, the two sequences have the same limit. Since $\log w /(w-1) \rightarrow 1$ as $w \rightarrow 1$, we can replace $\Sigma_{\nu_{n k}^{\circ}}(z)-1$ by $\log \Sigma_{\nu_{n k}^{\circ}}(z)$. The result follows by choosing $r_{n}=-\log \lambda_{n}-\sum_{k=1}^{k_{n}} \log b_{n k}$.

Theorem 4.3. For an infinitesimal array $\left\{\nu_{n k}\right\}_{n, k} \subset \mathcal{M}_{\mathbb{T}}^{\times}$and a sequence $\left\{\lambda_{n}\right\}_{n=1}^{\infty}$ $\subset \mathbb{T}$, the following assertions are equivalent:

(1) The sequence $\nu_{n 1} \otimes \nu_{n 2} \otimes \cdots \otimes \nu_{n k_{n}} \otimes \delta_{\lambda_{n}}$ converges weakly to $\nu_{\square}^{\gamma, \sigma}$. 
(2) The sequence of measures

$$
d \sigma_{n}(t)=\sum_{k=1}^{k_{n}}(1-\Re t) d \nu_{n k}^{\circ}(t)
$$

converges weakly on $\mathbb{T}$ to $\sigma$, and the limit

$$
\lim _{n \rightarrow \infty} e^{i \gamma_{n}}=e^{i \gamma}
$$

exists, where

$$
\gamma_{n}=\arg \lambda_{n}+\sum_{k=1}^{k_{n}}\left[\int_{\mathbb{T}} \Im t d \nu_{n k}^{\circ}(t)+\arg b_{n k}\right] .
$$

Proof. Assume (1) holds. From Theorem 2.1, there exists $\varepsilon \in(0,1)$ such that all $\Sigma_{\nu_{n k}}$ are defined in $K_{\varepsilon}^{\prime}=\{z /(1+z):|z| \leq \varepsilon\}$, and we have

$$
\lim _{n \rightarrow \infty}\left(\frac{1}{\lambda_{n}} \prod_{k=1}^{k_{n}} \Sigma_{\nu_{n k}}(z)\right)=\Sigma_{\nu_{\bigotimes}^{\gamma, \sigma}}(z)=e^{u_{\gamma, \sigma}(z)}
$$

uniformly on $K_{\varepsilon}^{\prime}$. Hence, by Lemma 4.2 and the definition of $u_{\gamma, \sigma}(z)$, we have

$$
\lim _{n \rightarrow \infty} \exp \left(-\log \lambda_{n}+\sum_{k=1}^{k_{n}}\left[h_{n k}(z)-\log b_{n k}\right]\right)=\exp \left(-i \gamma+\int_{\mathbb{T}}\left[\frac{1+t z}{1-t z}\right] d \sigma(t)\right)
$$

uniformly on $K_{\varepsilon}^{\prime}$. Taking the absolute value on both sides of (4.1), we deduce that

$$
\lim _{n \rightarrow \infty} \exp \left(\Re\left[\sum_{k=1}^{k_{n}} h_{n k}(z)\right]\right)=\exp \left(\int_{\mathbb{T}} \Re\left[\frac{1+t z}{1-t z}\right] d \sigma(t)\right)
$$

uniformly on $K_{\varepsilon}^{\prime}$. Note that

$$
-\log \lambda_{n}+\sum_{k=1}^{k_{n}}\left[h_{n k}(z)-\log b_{n k}\right]=-i \gamma_{n}+\int_{\mathbb{T}}\left[\frac{1+t z}{1-t z}\right] d \sigma_{n}(t) .
$$

Moreover, the real part of the function $-\log \lambda_{n}+\sum_{k=1}^{k_{n}}\left[h_{n k}(z)-\log b_{n k}\right]$ is the Poisson integral of the measure $d \sigma_{n}\left(\frac{1}{t}\right)$, and hence (4.2) uniquely determines the weak cluster point of $\left\{\sigma_{n}\right\}_{n=1}^{\infty}$ which is $\sigma$. We therefore conclude the weak convergence of the sequence $\left\{\sigma_{n}\right\}_{n=1}^{\infty}$. Moreover, consider $z=0$ in (4.1) and (4.2) to deduce that

as desired.

$$
\lim _{n \rightarrow \infty} \frac{e^{i \gamma}}{e^{i \gamma_{n}}}=1
$$

The converse implication is fairly easy now, since one can basically reverse the steps to reach the statement (1) by using Lemma 4.2 and the fact that $\left\{-\log \lambda_{n}+\right.$ $\left.\sum_{k=1}^{k_{n}}\left[h_{n k}(z)-\log b_{n k}\right]\right\}_{n=1}^{\infty}$ is normal in $\mathbb{D}$. Therefore the details of the proof are left to the reader.

The previous result does not cover the possibility that the measures $\nu_{n 1} \otimes \nu_{n 2} \otimes$ $\cdots \otimes \nu_{n k_{n}} \otimes \delta_{\lambda_{n}}$ might converge to the Haar measure $m$. We address now this special case. Let us also note for further use the equality

$$
\Sigma_{\nu}(0)=\frac{1}{\int_{\mathbb{T}} t d \nu(t)}, \quad \nu \in \mathcal{M}_{\mathbb{T}}^{\times}
$$


Theorem 4.4. For an infinitesimal array $\left\{\nu_{n k}\right\}_{n, k} \subset \mathcal{M}_{\mathbb{T}}^{\times}$and a sequence $\left\{\lambda_{n}\right\}_{n=1}^{\infty}$ $\subset \mathbb{T}$, the following assertions are equivalent:

(1) The sequence $\nu_{n 1} \otimes \nu_{n 2} \otimes \cdots \otimes \nu_{n k_{n}} \otimes \delta_{\lambda_{n}}$ converges weakly to $m$.

$$
\lim _{n \rightarrow \infty} \sum_{k=1}^{k_{n}} \int_{\mathbb{T}}(1-\Re t) d \nu_{n k}^{\circ}(t)=+\infty .
$$

Proof. Assume (2) holds. Define

$$
\nu_{n}=\nu_{n 1} \otimes \nu_{n 2} \otimes \cdots \otimes \nu_{n k_{n}} \otimes \delta_{\lambda_{n}}, \quad n \in \mathbb{N} .
$$

The compactness of $\mathbb{T}$ implies that $\left\{\nu_{n}\right\}_{n=1}^{\infty}$ is tight. Suppose $\nu$ is a weak cluster point of $\left\{\nu_{n}\right\}_{n=1}^{\infty}$. From the free multiplicative analogue of Hinčin's theorem (Theorem 2.1 in [2]), the measure $\nu$ is $\otimes$-infinitely divisible. By passing to a subsequence, we may assume that $\nu_{n}$ converges weakly to $\nu$ as $n \rightarrow \infty$. By (4.3), we can reformulate the statement (2) as follows:

$$
\lim _{n \rightarrow \infty} \Re \sum_{k=1}^{k_{n}} h_{n k}(0)=+\infty .
$$

Then the inequality (2.2) implies that

$$
\lim _{n \rightarrow \infty} \Re \sum_{k=1}^{k_{n}} \log \Sigma_{\nu_{n k}}(0)=+\infty,
$$

and consequently we deduce that

$$
\begin{aligned}
\left|\int_{\mathbb{T}} t d \nu(t)\right| & =\lim _{n \rightarrow \infty}\left|\int_{\mathbb{T}} t d \nu_{n}(t)\right| \\
& =\lim _{n \rightarrow \infty} \frac{1}{\left|\prod_{k=1}^{k_{n}} \Sigma_{\nu_{n k}}(0)\right|} \\
& =\lim _{n \rightarrow \infty} \exp \left(-\Re \sum_{k=1}^{k_{n}} \log \Sigma_{\nu_{n k}}(0)\right)=0 .
\end{aligned}
$$

Therefore, the $\otimes$-infinitely divisible measure $\nu$ has zero first moment, and hence we conclude that $\nu=m$. Moreover, the full sequence $\nu_{n}$ converges weakly to $m$ since $\left\{\nu_{n}\right\}_{n=1}^{\infty}$ has a unique weak cluster point $m$.

Conversely, assume (1) holds but (2) fails to be true. By passing, if necessary, to a subsequence, we may assume the sequence of measures

$$
d \sigma_{n}(t)=\sum_{k=1}^{k_{n}}(1-\Re t) d \nu_{n k}^{\circ}(t)
$$

converges weakly to a finite positive Borel measure $\sigma$ on $\mathbb{T}$. Since the sequence of functions $\left\{-\log \lambda_{n}+\sum_{k=1}^{k_{n}}\left[h_{n k}(z)-\log b_{n k}\right]\right\}_{n=1}^{\infty}$ is normal in $\mathbb{D}$, we may assume, by passing to a further subsequence, that

$$
\lim _{n \rightarrow \infty}\left(-\log \lambda_{n}+\sum_{k=1}^{k_{n}}\left[h_{n k}(z)-\log b_{n k}\right]\right)=f(z)
$$


uniformly on compact subsets of $\mathbb{D}$, where the function $f$ is analytic in $\mathbb{D}$. Note that the function $f$ is not identically infinity since

$$
\Re f(z)=\lim _{n \rightarrow \infty} \int_{\mathbb{T}} \Re\left[\frac{1+t z}{1-t z}\right] d \sigma_{n}(t)=\int_{\mathbb{T}} \Re\left[\frac{1+t z}{1-t z}\right] d \sigma(t), \quad z \in \mathbb{D} .
$$

Then, as in the proof of Theorem 4.3, we conclude that there exists $\gamma \in \mathbb{R}$ such that

$$
\lim _{n \rightarrow \infty} e^{i \gamma_{n}}=e^{i \gamma}
$$

where the number $\gamma_{n}$ is defined as in Theorem 4.3. An application of Theorem 4.3 then shows that a subsequence of $\left\{\nu_{n}\right\}_{n=1}^{\infty}$ converges weakly to $\nu_{\unrhd}^{\gamma, \sigma}$, which contradicts (1). Therefore (2) must be true.

\section{REFERENCES}

[1] S. T. Belinschi and H. Bercovici, Atoms and regularity for measures in a partially defined free convolution semigroup, Math. Z. 248 (2004), no. 4, 665-674. MR2103535 (2006i:46095)

[2] Hinčin's theorem for multiplicative free convolution, Canadian Math. Bulletin, 51 (2008), no. 1, 26-31.

[3] H. Bercovici and V. Pata, Stable laws and domain of attraction in free probability, with an appendix by Ph. Biane, Ann. Math. 149 (1999), 1023-1060. MR1709310 (2000i:46061)

[4] Limit laws for products of free and independent random variables, Studia Math. 141 (2000), no. 1, 43-52. MR1782911 (2001i:46105)

[5] H. Bercovici and D. V. Voiculescu, Lévy-Hinčin type theorems for multiplicative and additive free convolution, Pacific J. Math. 153 (1992), no. 2, 217-248. MR.1151559 (93k:46052)

[6] _ Free convolution of measures with unbounded support, Indiana Univ. Math. J. 42 (1993), no. 3, 733-773. MR1254116 (95c:46109)

[7] Ph. Biane, Processes with free increments, Math. Z. 227 (1995), no. 1, 143-174. MR1605393 (99e:46085)

[8] G. P. Chistyakov and F. Götze, The arithmetic of distributions in free probability, Arxiv: math. PS/0508245.

[9] L Limit theorems in free probability. I, Ann. Probab. 36 (2008), no. 1, 54-90. MR2370598

[10] D. V. Voiculescu, Multiplication of certain noncommuting random variables, J. Operator Theory 18 (1987), 223-235. MR915507(89b:46076)

[11] - The analogues of entropy and of Fisher's information measure in free probability. I, Comm. Math. Phys. 155 (1993), no. 1, 71-92. MR.1228526 (94k:46137)

[12] - The coalgebra of the free difference quotient and free probability, Int. Math. Res. Not. 2000 (2000), no. 2, 79-106. MR.1744647 (2001d:46096)

[13] _ Analytic subordination consequences of free Markovianity, Indiana Univ. Math. J. 51 (2002), no. 5, 1161-1166. MR1947871 (2003k:46100)

Department of Mathematics, Indiana University, Bloomington, Indiana 47405-4301

E-mail address: bercovic@indiana.edu

Department of Mathematics, Indiana University, Bloomington, Indiana 47405-4301

E-mail address: jiuwang@indiana.edu 